\title{
KINETICS OF HEAT INACTIVATION OF PHENYL VALERATE HYDROLASES FROM HEN AND RAT BRAIN*
}

\author{
Elsa Reiner, $†$ Cinda S. Davis, $\ddagger$ Bradley W. Schwab,§ LaWrence M. Schopfer $\|$ \\ and RUDY J. RICHARDSON \\ Neurotoxicology Research Laboratory, Toxicology Program, School of Public Health, and |Department of Biological \\ Chemistry, School of Medicine, The University of Michigan, Ann Arbor, MI 48109, U.S.A.
}

(Received 10 July 1986; accepted 17 February 1987)

\begin{abstract}
Heat inactivation was studied at $45,50,55$, and $60^{\circ}$ for all of the phenyl valerate hydrolases (PVase), including neurotoxic esterase (NTE) and inhibitor-resistant esterase (IRE), in homogenates of hen or rat brain or in preparations of hen brain microsomal membranes. Hen and rat brain homogenates were prepared in buffer $\left(50 \mathrm{mM}\right.$ Tris $/ 0.20 \mathrm{mM}$ EDTA, pH 8.00 , at $\left.25^{\circ}\right)$. Hen brain microsomes were suspended either in buffer or in aqueous dimethyl sulfoxide (DMSO, 40\%, w/v), or solubilized either in aqueous Triton X-100 $\left(0.10 \%\right.$, w/v) or in $40 \%$ (w/v) DMSO. Enzyme activities were measured at $37^{\circ}$ using phenyl valerate as substrate. Each enzyme activity in all of the preparations exhibited biphasic heat inactivation kinetics. Apparent rate constants were calculated for the fast $\left(k_{f}\right)$ and slow $\left(k_{s}\right)$ reactions, along with the relative amounts of activity in each component $\left(A_{f}, A_{s}\right)$ expressed as percentages of the total activity. For a given preparation and temperature, respective values of $k_{f}$ or $k_{s}$ were similar for PVase, NTE, and IRE, with a mean $k_{f} / k_{s}$ ratio of 52 across all preparations. $A_{f}$ and $A_{s}$ were a function of temperature. Mean values of the apparent activation energies $\left(E_{a}\right)$ for all activities and preparations were 44 and $25 \mathrm{kcal} / \mathrm{mol}$ for the fast and slow inactivation reactions respectively. These results indicate that all phenyl valerate hydrolases in hen and rat brain undergo a common heat-induced structural change leading to loss of enzymic activity.
\end{abstract}

The objective of this work was to differentiate by heat inactivation the enzymes that hydrolyze phenyl valerate in hen and rat brain. These enzymes belong to the general class of carboxylic ester hydrolases (EC 3.1.1.1) $[1,2]$. Within this group, $\mathrm{NTE}^{* *}$ is of particular toxicological interest due to its putative role in the development of neuropathy caused by certain OPs [3-5].

In hens, inhibition of $>70 \%$ of brain NTE by OPs capable of undergoing aging (time-dependent scission of a side chain from the phosphoryl moiety) is correlated with subsequent development of clinical and histopathological evidence of neuropathy $[4,5]$. Rat brain possesses NTE activity that is similar to hen

*Presented in part at the 25th Annual Meeting of the Society of Toxicology, New Orleans, LA, March 3-7, 1986.

†Visiting Professor of Toxicology on leave from the Institute of Medical Research and Occupational Health, Zagreb, Yugoslavia.

$¥$ Present address: Director, Women in Science Program, Center for the Continuing Education of Women, The University of Michigan, Ann Arbor, MI.

\$Present address: ERT, Inc., Concord, MA.

ISend correspondence to: Dr. Rudy J. Richardson, Neurotoxicology Research Laboratory, Toxicology Program, M-7525 School of Public Health-II, The University of Michigan, Ann Arbor, MI 48109, U.S.A.

**Abbreviations: NTE, neurotoxic esterase; OP(s), organophosphorus compound(s); mipafox, $N, N^{\prime}$-di-2propyl phosphorodiamidofluoridate; DMSO, dimethyl sulfoxide; PVase, total phenyl valerate hydrolases; IRE, inhibitor resistant esterase; and paraoxon, diethyl 4nitrophenyl phosphate. brain NTE with respect to $\mathrm{pH}$ profile, mipafox inhibition, and molecular weight of the organophosphorylated subunit [6]. Inhibition of $>65 \%$ of rat brain NTE results in spinal cord lesions, but clinical signs of neuropathy are not evident [7].

Previous work in our laboratory indicated that hen brain NTE is more thermostable than rat brain NTE and that the kinetics of inactivation are biphasic in both cases [8]. The suggestion was made that this could indicate two enzymes/forms of NTE in each preparation. The presence of two enzymes/forms of NTE has also been suggested from the kinetics of inhibition by OPs $[9,10]$. We have now undertaken to study the effect of heat in more detail and have included the whole group of phenyl valerate hydrolases, of which NTE is only one subgroup.

\section{MATERIALS AND METHODS}

Chemicals. The buffer used in all experiments was $50 \mathrm{mM}$ Tris $/ 0.20 \mathrm{mM}$ EDTA, $\mathrm{pH} 8.00$ (pH at $25^{\circ}$ ). All aqueous solutions were prepared with deionizeddistilled water. Commercially obtained chemicals were analytical reagent grade or the highest grade available. Mipafox and phenyl valerate were synthesized and purified as described by Johnson [11].

Animals. Male CD rats, $250-300 \mathrm{~g}$ (Charles River, Portage, MI), or adult (>1 yr old) White Leghorn laying hens (Omega Chicks, Haslett, MI) were housed four per cage in separate air-conditioned rooms $\left(21-24^{\circ}\right)$ with an automatic 12-hr light/dark cycle. Rats received Purina Rat Chow and tap water ad lib.; hens received Purina Layena laying mash and tap water ad lib. 
Rat and hen brain homogenates. Animals were killed by decapitation. Brain homogenates $(10 \%$, w/v) were prepared in ice-cold buffer using a glass/Teflon homogenizer $(0.25 \mathrm{~mm}$ clearance, 15 strokes at $2300 \mathrm{rpm}$ ).

Hen brain microsomal membranes in buffer or $D M S O$. Brains were homogenized in water, and a microsomal pellet $(100,000 \mathrm{~g}, 60 \mathrm{~min})$ was prepared by differential centrifugation. The microsomal pellet derived from $3.00 \mathrm{~g}$ of brain was suspended in $20.0 \mathrm{ml}$ buffer or aqueous $40 \%$ (w/v) DMSO.

Solubilized microsomal membranes. The microsomal pellet derived from $3.00 \mathrm{~g}$ of brain was rehomogenized in $20.0 \mathrm{ml}$ of aqueous $0.10 \%(\mathrm{w} / \mathrm{v})$ Triton X-100 or $40 \%$ (w/v) DMSO and centrifuged at $100,000 \mathrm{~g}$ for $60 \mathrm{~min}$. The Triton supernatant fraction was freeze-dried and kept at $-20^{\circ}$ until use, at which time the preparation was dissolved in buffer; DMSO supernatant fractions were prepared immediately before use.

Heat inactivation. Aliquots $(0.30 \mathrm{ml})$ of the enzyme preparations were transferred to $13 \times 100 \mathrm{~mm}$ glass culture tubes that had been prewarmed to the inactivation temperature $\left(45,50,55\right.$, or $\left.60^{\circ}\right)$ and allowed to incubate in a constant-temperature water bath for a set interval in the range 0-120 min before adding ice-cold buffer $(4.70 \mathrm{ml}$ for whole brain homogenates; $2.7 \mathrm{ml}$ for microsomal preparations). The tubes were then placed on ice until being assayed for residual phenyl valerate hydrolase activities. The mean protein content $(\mathrm{mg} / \mathrm{ml})$ during heat inactivation was 5.2 for hen or rat brain homogenates; 0.17 for hen brain microsomes in buffer or DMSO; 1.8 for Triton-solubilized microsomes; and 0.31 for DMSOsolubilized microsomes.

Assay of phenyl valerate hydrolase activities. PVase, NTE, and IRE activities, as previously defined $[12,13]$, were determined by a modification [14] of the method of Johnson for NTE [11], adjusting the preincubation conditions as appropriate to select the activity of interest. PVase activity was determined from the ratc of phenyl valerate hydrolysis when the enzyme preparation was not preincubated with either paraoxon or mipafox. IRE activity was determined from the rate of phenyl valerate hydrolysis when the enzyme preparation was preincubated $(20 \mathrm{~min})$ with paraoxon $(100 \mu \mathrm{M})$ and mipafox $(50 \mu \mathrm{M})$. NTE activity was determined from the difference between rates of phenyl valerate hydrolysis in two aliquots of a given enzyme preparation of which one was preincubated (20 min) only with paraoxon $(100 \mu \mathrm{M})$ and the other with paraoxon $(100 \mu \mathrm{M})$ and mipafox $(50 \mu \mathrm{M})$. All enzyme activities were measured at $37^{\circ}$ with phenyl valerate as substrate. The final substrate concentration during enzyme assay was $1.35 \mathrm{mM}$. The phenol produced during substrate hydrolysis (15 or $30 \mathrm{~min}$ ) was reacted with 4-aminoantipyrine and $\mathrm{K}_{3} \mathrm{Fe}(\mathrm{CN})_{6}$, and the colored product was measured spectrophotometrically at $510 \mathrm{~nm}$. All activities were corrected for the non-enzymic hydrolysis of phenyl valerate. Details about the preparation of reagents, including substrate and inhibitors, have been described previously [11-14].

Protein assay. The Bio-Rad protein assay kit (BioRad Laboratories, Richmond, CA) based on the dyebinding method of Bradford [15] was used. Bovine serum albumin, fraction V (Sigma Chemical Co., St Louis, MO), was used as a reference standard.

Kinetic constants. Plots of $\log A$ versus $t$, where $A=$ percent remaining activity and $t=$ time (min), were resolved into two linear components by the method of residuals $[1,16]$ (see Fig. 1). Apparent rate constants for the fast $\left(k_{f}\right)$ and slow $\left(k_{s}\right)$ reactions were calculated from the slopes, and relative amounts of activity in the fast $\left(A_{f}\right)$ and slow $\left(A_{s}\right)$ components were determined from the intercepts of cach linc on the ordinate. No rate constants were calculated when $A_{f}$ or $A_{s}$ was $\leqslant 10 \%$ of the total activity.

Activation energies. Apparent activation energies $\left(E_{a}\right)$ for heat inactivation were determined from the slopes of the Arrhenius plots [17] for each of the activities of interest.

\section{RESULTS}

The specific activities of the phenyl valerate hydrolases in the preparations studied are shown in Table 1. Hen brain homogenates contained more PVase

Table 1. Distribution of phenyl valerate hydrolase activities in hen and rat brain preparations

\begin{tabular}{lcccc}
\hline Preparation & $\begin{array}{c}\text { PVase } \\
\left(\mathrm{nmol} \cdot \mathrm{min}^{-1} \cdot(\mathrm{mg} \text { protein })^{-1}\right)\end{array}$ \\
\hline $\begin{array}{c}\text { Hen brain } \\
\text { Homogenate (13) }\end{array}$ & $335 \pm 9$ & $60.3 \pm 1.3$ & $21.9 \pm 0.7$ \\
$\begin{array}{c}\text { Microsomes suspended } \\
\text { in buffer (3) }\end{array}$ & $421 \pm 12$ & $168 \pm 4$ & 26 & \pm 1 \\
$\quad \begin{array}{c}\text { Microsomes suspended } \\
\text { in DMSO (4) }\end{array}$ & $417 \pm 16$ & $162 \pm 8$ & 27 & \pm 2 \\
$\begin{array}{c}\text { Triton-solubilized } \\
\text { microsomes (4) }\end{array}$ & $224 \pm 2$ & $38 \pm 5$ & $6.7 \pm 0.7$ \\
$\begin{array}{c}\text { DMSO-solubilized } \\
\text { microsomes (4) }\end{array}$ & $219 \pm 8$ & $50 \pm 4$ & $13 \pm 1$ \\
$\begin{array}{c}\text { Rat brain } \\
\text { Homogenate (10) }\end{array}$ & $199 \pm 5$ & $23.9 \pm 0.8$ & $25.9 \pm 0.6$ \\
\hline
\end{tabular}

Data are mean values $\pm S E$; the numbers in parentheses are the number of experiments. 
and NTE than rat brain homogenates, whereas the specific activities of IRE were about the same. NTE and IRE were equally active in rat brain homogenate, whereas in hen brain homogenate NTE was about 2.8 times more active than IRE. Compared with homogenate, hen brain microsomes suspended in buffer or DMSO were enriched in PVase and NTE, but not in IRE.

The kinetics of heat inactivation of all phenyl valerate hydrolases were qualitatively the same: at all temperatures the reaction was biphasic. An example of the time course of heat inactivation is shown in Fig. $1 \mathrm{a}$ for the inactivation of PVase in hen brain homogenates. The reaction can be described by the sum of two first-order exponential terms as shown in Fig. $1 \mathrm{~b}$. The kinetic constants derived from Fig. 1b were used to calculate the solid curve in Fig. 1a. The calculated curves and the experimental points agreed well for all studied phenyl valerate hydrolases, and this served as a verification of the validity of the evaluation of the kinetic constants.

The kinetic constants evaluated for the phenyl valerate hydrolases in hen and rat brain homogenates are listed in Tables 2 and 3. For a given preparation and temperatue, the activities of PVase, NTE and IRE decreased at nearly the same rate, and the relative amounts of activities in the fast and slow components $\left(A_{f}\right.$ and $A_{s}$ ) were similar for all three groups of enzymes. The $k_{f} / k_{s}$ ratio was in all cases large, with a mean value of 52 across the two preparations. An overall comparison of the rate constants from rat brain with those from hen brain indicates that phenyl valerate hydrolases tend to inactivate faster in rat brain homogenates than in hen brain homogenates.

Phenyl valerate hydrolases are membrane-bound proteins $[2,12]$ that may be solubilized in detergents
Table 2. Kinetic constants for heat inactivation of PVase, NTE, and IRE in hen brain homogenate

\begin{tabular}{|c|c|c|c|c|c|}
\hline \multirow[b]{2}{*}{ Enzyme } & \multirow[b]{2}{*}{$t\left({ }^{\circ} \mathrm{C}\right)$} & \multicolumn{2}{|c|}{ Fast reaction } & \multicolumn{2}{|c|}{ Slow reaction } \\
\hline & & $k_{f}\left(\min ^{-1}\right)$ & $A_{f}(\%)$ & $k_{s}\left(\min ^{-1}\right)$ & $A_{s}(\%)$ \\
\hline pVase (3) & 60 & 0.64 & 88 & 0.0050 & 12 \\
\hline PVase (3) & 55 & 0.18 & 65 & 0.0047 & 35 \\
\hline PVase (3) & 50 & 0.068 & 20 & 0.0040 & 80 \\
\hline NTE (3) & 60 & 0.68 & 90 & 工 & 10 \\
\hline NTE (5) & 55 & 0.10 & 52 & 0.0059 & 48 \\
\hline NTE (4) & 50 & - & 0 & 0.0034 & 100 \\
\hline IRE & 60 & 1.10 & 90 & & 10 \\
\hline IRE (3) & 55 & 0.15 & 65 & 0.0 & 35 \\
\hline IRE (4) & 50 & 0.11 & 37 & 0.0043 & 63 \\
\hline
\end{tabular}

Hen brain homogenates in buffer were incubated for fixed times in the range $0-120 \mathrm{~min}$ at the temperatures indicated. Data from the number of experiments shown in parentheses were plotted as $\log$ (percent remaining activity) versus time for each phenyl valerate hydrolase and inactivation temperature (cf. Fig. 1a). Apparent rate constants for the fast $\left(k_{f}\right)$ and slow $\left(k_{s}\right)$ inactivation reactions were determined graphically from the slopes of the resolved linear components of the biphasic curves; the relative amounts of activity inactivating at the fast $\left(A_{f}\right)$ and slow $\left(A_{s}\right)$ rates were determined from the intercepts of the linear components on the ordinate (cf. Fig. 1b).

$[2,18,19]$ or organic solvents [14] with partial retention of enzymic activity. To determine whether the biphasic kinetics observed in crude homogenates may have been due to a membrane effect, heat inactivation was studied in preparations of hen brain microsomes solubilized in Triton X-100 or DMSO. An example of the heat inactivation of NTE in DMSO-solubilized hen brain microsomes is shown in
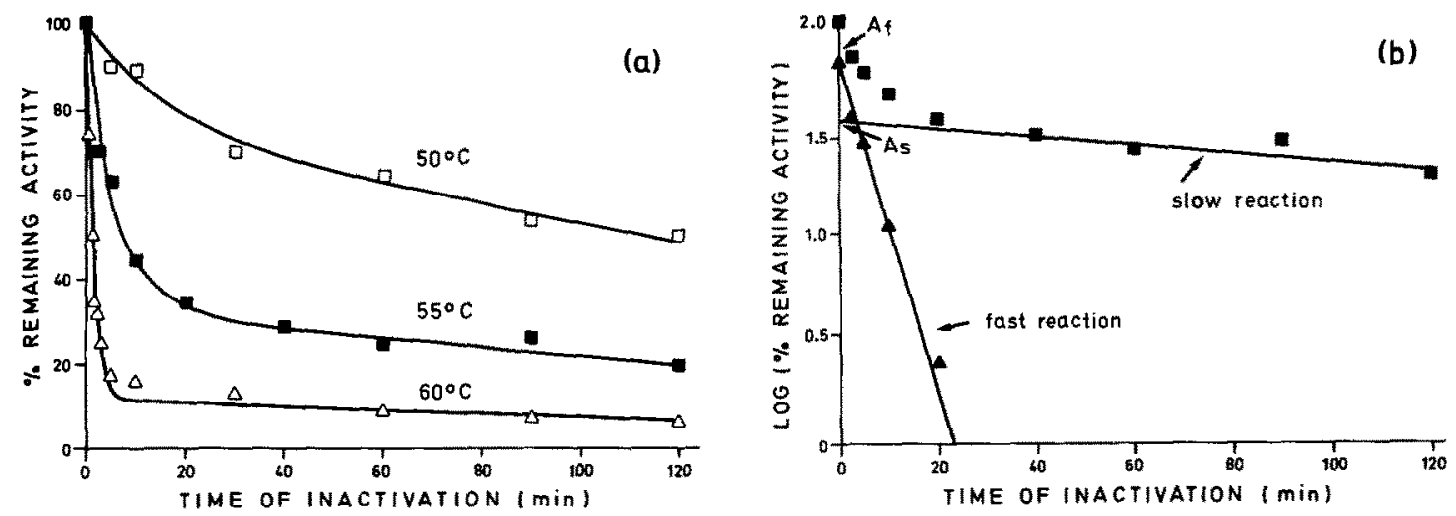

Fig. 1. Heat inactivation of PVase in hen brain homogenate. (a) Plot of percent remaining activity versus time of incubation at $50(\square), 55(\square)$, and $60^{\circ}(\triangle)$. Each point is the mean value obtained from three experiments. The solid lines were calculated from the equation:

$$
A=A_{f} \cdot e^{-k f \cdot t}+A_{s} \cdot e^{-k_{s} \cdot t}
$$

where $A=$ percent remaining activity, $k_{f}=$ apparent rate constant of inactivation for the fast reaction, $k_{s}$ $=$ apparent rate constant of inactivation for the slow reaction, $A_{f}=$ relative amount of activity $(\%)$ inactivating at the fast rate, $A_{s}=$ relative amount of activity inactivating at the slow rate, and $t=$ time (min). (b) Plot of $\log A$ versus time for heat inactivation at $55^{\circ}$, showing the graphical method described in Rets. 1 and 16 for obtaining the apparent rate constants and relative activities from the slopes and intercepts of the lines. The solid squares (D) are the experimental points. The solid triangles (A) are calculated values obtained by subtracting the slow component of the inactivation curve from the initial (fast) component of the curve. 
Table 3. Kinetic constants for heat inactivation of PVase, NTE, and IRE in rat brain homogenates

\begin{tabular}{lcccccc}
\hline & & \multicolumn{2}{c}{ Fast reaction } & & \multicolumn{2}{c}{ Slow reaction } \\
\cline { 7 - 7 } \cline { 5 - 6 } Enzyme & $t\left({ }^{\circ} \mathrm{C}\right)$ & $k_{f}\left(\mathrm{~min}^{-1}\right)$ & $A_{f}(\%)$ & $k_{s}\left(\mathrm{~min}^{-1}\right)$ & $A_{s}(\%)$ \\
\hline PVase (3) & 55 & 0.42 & 75 & 0.013 & 25 \\
PVase (2) & 50 & 0.26 & 37 & 0.0061 & 63 \\
PVase (2) & 45 & 0.11 & 26 & 0.0020 & 74 \\
NTE (4) & 55 & 0.98 & 100 & & 0 \\
NTE (3) & 50 & 0.15 & 73 & 0.0062 & 27 \\
NTE (2) & 45 & - & 0 & 0.0040 & 100 \\
IRE (4) & 55 & 0.85 & 85 & 0.0041 & 15 \\
IRE (3) & 50 & 0.29 & 62 & 0.0069 & 38 \\
IRE (2) & 45 & 0.12 & 27 & 0.0062 & 73 \\
\hline
\end{tabular}

Rat brain homogenates in buffer were incubated for fixed times in the range $0-120 \mathrm{~min}$ at the temperatures indicated. Data from the number of experiments shown in parentheses were plotted as log (percent remaining activity) versus time for each phenyl valerate hydrolase and inactivation temperature. Apparent rate constants for the fast $\left(k_{f}\right)$ and slow $\left(k_{s}\right)$ inactivation reactions and the relative amounts of activity inactivating at the fast $\left(A_{f}\right)$ and slow $\left(A_{s}\right)$ rates were determined as described in Table 2 .

Fig. 2. Again, the reaction was biphasic. The same was true for PVase. A biphasic reaction was also observed with all of the other microsomal preparations studied for both PVase and NTE activities, as can be seen from the kinetic constants determined for these reactions listed in Table 4 . The $k_{f} / k_{s}$ ratio was large, with a mean value of 51 for PVase and NTE activities in all microsomal preparations. Data are lacking for heat inactivation of IRE, because these activities were too low in all microsomal preparations to allow meaningful determinations to be made. A quantitative comparison between the rate constants of inactivation in microsomal preparations (Table 4) with those in total brain homogenates (Table 2) would not be justified, because the protein content during heat inactivation was different in the two preparations (see Materials and Methods), and the

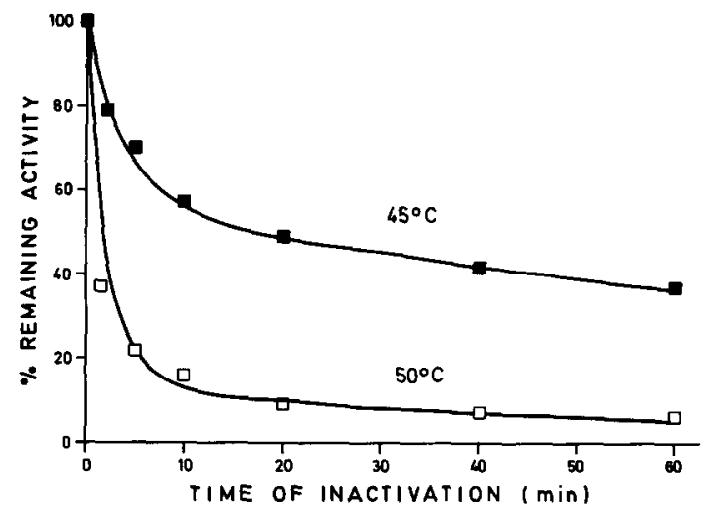

Fig. 2. Heat inactivation of NTE in hen brain microsomes solubilized in aqueous $40 \%$ (w/v) DMSO. Plot of percent remaining activity versus time of incubation at $45(\square)$ and $50^{\circ}(\square)$. Each point is the mean value from two experiments. The solid curves were calculated as described under Fig. 1.
Table 4. Kinetic constants for heat inactivation of PVase and NTE in hen brain microsomal preparations

\begin{tabular}{|c|c|c|}
\hline & Fast reaction & Slow reaction \\
\hline $\begin{array}{l}\text { Preparation/ } \\
\text { Enzyme } \quad t\left({ }^{\circ} \mathrm{C}\right)\end{array}$ & $k_{f}\left(\min ^{-1}\right) \quad A_{f}(\%)$ & $k_{s}\left(\min ^{-1}\right) \quad A_{f}(\%)$ \\
\hline
\end{tabular}

Microsomes

in buffer/

$\begin{array}{llllll}\text { PVase } & 50 & 0.24 & 34 & 0.0058 & 66 \\ \text { PVase } & 45 & 0.36 & 18 & 0.0033 & 82 \\ \text { NTE } & 50 & 0.30 & 20 & 0.0030 & 80 \\ \text { NTE } & 45 & - & 10 & * & 90\end{array}$

Microsomes

in DMSO/

\begin{tabular}{llllll} 
PVase & $\mathbf{5 0}$ & 0.40 & 77 & 0.0071 & 23 \\
PVase & $\mathbf{4 5}$ & $\mathbf{0 . 2 5}$ & 49 & 0.0049 & 51 \\
NTE & 50 & 0.34 & 80 & 0.0069 & 20 \\
NTE & 45 & 0.12 & 44 & 0.0019 & 56 \\
\hline
\end{tabular}

Microsomes

solubilized

in Triton

$\begin{array}{llllll}\text { PVase } & 50 & 0.32 & 50 & 0.0108 & 50 \\ \text { PVase } & 45 & 0.12 & 24 & 0.0058 & 76 \\ \text { NTE } & 50 & 0.38 & 80 & 0.0210 & 20 \\ \text { NTE } & 45 & 0.22 & 53 & 0.0086 & 47\end{array}$

Microsomes

solubilized

in DMSO/

$\begin{array}{llllll}\text { PVase } & 50 & 1.10 & 78 & 0.0110 & 22 \\ \text { PVase } & 45 & 0.34 & 58 & 0.0080 & 42 \\ \text { NTE } & 50 & 0.44 & 86 & 0.0160 & 14 \\ \text { NTE } & 45 & 0.25 & 44 & 0.0069 & 56\end{array}$

Hen brain microsomal preparations were incubated for fixed times in the range $0-60 \mathrm{~min}$ at the indicated temperatures. Data from two experiments were plotted as log (percent remaining activity) versus time of inactivation for PVase and NTE in each preparation. Apparent rate constants for the fast $\left(k_{f}\right)$ and slow $\left(k_{s}\right)$ inactivation reactions and the relative amounts of activity inactivating in the fast $\left(A_{f}\right)$ and slow $\left(A_{s}\right)$ reactions were determined as described in Table 2.

*Activity was stable between 5 and $60 \mathrm{~min}$.

actual rates are likely to depend on the protein content.

Apparent activation energies $\left(E_{a}\right)$ for the fast and slow heat inactivation reactions of each phenyl valerate hydrolase studied in the various preparations were calculated from the data in Tables $2-4$. The mean $E_{a}$ values throughout all preparations were 44 and $25 \mathrm{kcal} / \mathrm{mol}$ for the fast and slow inactivation reactions respectively.

\section{DISCUSSION}

The kinetics of heat inactivation revealed a striking qualitative similarity between all phenyl valerate hydrolases in preparations of hen and rat brain. The activities of the total PVase and of the two subgroups, NTE and IRE, inactivated in a biphasic manner. For a given preparation and temperature, the activities of PVase, NTE and IRE inactivated almost at the same rates, and the relative amounts of activities in the fast 
and slow components were similar. The large magnitude of the apparent activation energies is characteristic of heat denaturation of proteins [20] and was observed for both the fast and slow reactions. These results are consistent with the existence of structural features in common among the phenyl valerate hydrolases enabling these enzymes to undergo similar heat-induced structural change leading to loss in enzymic activity through the formation of at least one enzymically active intermediate $[21,22]$. The earlier observation that rat brain NTE is more thermally labile than hen brain NTE [8] was substantiated and extended to the other phenyl valerate hydrolases in the present study, and is in agreement with another recent report [23].

Phenyl valerate hydrolases are membrane-bound enzymes [2,12]. DMSO is a powerful disruptor of membrane structure [24] that has been shown recently to solubilize active NTE [14]. Triton X-100 has also been shown to solubilize active NTE [18, 19], but this detergent would be expected to yield a micellar structure capable of mimicking the lipid environment of the original membrane [25]. Suspending microsomes in buffer does not solubilize NTE $[14,18,19]$ and should leave the membrane intact. The fact that the kinetics of heat inactivation were qualitatively similar in these different preparations leads to the conclusion that the biphasic reaction is not due to a membrane effect.

The biphasic kinetics of heat inactivation have been observed earlier for the inactivation of NTE from rat and hen brain homogenates $[8,9]$. One interpretation of these results is that there are two forms of NTE initially present in each preparation that differ in thermal stability [26]; two forms of NTE have also been inferred from analyses of inhibition kinetics $[9,10]$. Considering the results described in this paper, the dual-form interpretation would seem to be no longer justified, for the following reasons. First, the total PVase activity (which clearly contains several enzymes) showed the same kinetics of inactivation as the subgroups NTE and IRE, and at a given temperature the relative activities $A_{f}$ and $A_{s}$ (representing the amounts of activity which inactivate at the fast and slow rates respectively) were similar for PVase, NTE and IRE. It is very unlikely that in each group two enzymes would be present in about the same proportions and would exhibit the same thermal kinetic properties. Furthermore, the relative activities $A_{f}$ and $A_{s}$ were highly temperature dependent. If these activities represented two enzymes initially present with intrinsically different rates of inactivation, their ratio would not be expected to vary with temperature [21]. Consequently, the suggestion that the biphasic kinetics of inactivation reflect the initial presence of two enzymes does not seem to hold, and the alternative model of heat inactivation proceeding through at least one enzymic intermediate is favored $[21,22]$. This interpretation is in accord with a recent kinetic study of mipafox inhibition of NTE in which it was concluded that the data were better explained on the basis of Michaelis complex formation between enzyme and inhibitor than by invoking the presence of two isoenzymes of NTE [27].

Acknowledgements-This work was supported by NIH Research Grants 2R01 ES01611 and 1R01 ES02770.

\section{REFERENCES}

1. J. M. Chemnitius and R. Zech, Molec. Pharmac. 23, 717 (1983)

2. J. M. Chemnitius and R. Zech, Int. J. Biochem. 16, 361 (1984).

3. M. K. Johnson, Biochem J. 114, 711 (1969).

4. M. K. Johnson, J. Neurochem. 23, 785 (1974).

5. C. S. Davis, M. K. Johnson and R. J. Richardson, in Neurotoxicity of Industrial and Commercial Chemicals (Ed. J. L. O'Donoghue), Vol. II, p. 1. CRC Press, Boca Raton (1985).

6. R. Novak and S. Padilla, Fund. appl. Toxic. 6, 464 (1986).

7. S. Padilla and B. Veronesi, Toxic. appl. Pharmac. 78, 78 (1985).

8. B. R. Dudek, Ph.D. Thesis, The University of Michigan, p. 78. University Microfilms International, Ann Arbor (1979).

9. R. J. Richardson and B. R. Dudek, in Pesticide Chemistry: Human Welfare and the Environment (Eds. J. Miyamoto and P. C. Kearney), Vol. 3, p. 491. Pergamon Press, Oxford (1983).

10. J. M. Chemnitius, K. H. Haselmeyer and R. Zech, Archs Toxic. 53, 235 (1983).

11. M. K. Johnson, Archs Toxic. 37, 113 (1977).

12. R. J. Richardson, C. S. Davis and M. K. Johnson, $J$. Neurochem. 32, 607 (1979).

13. B. R. Dudek and R. J. Richardson, Biochem. Pharmac. 31, 1117 (1982).

14. B. W. Schwab, C-S. G. Davis, P. H. Miller and R. J. Richardson, Biochem. biophys. Res. Commun. 132, 81 (1985).

15. M. M. Bradford, Analyt. Biochem. 72, 248 (1976).

16. M. Skrinjarič-Spoljar and E. Reiner, Biochim. biophys. Acta 165, 289 (1968).

17. K. J. Laidler, Reaction Kinetics, Vol. 1, p. 45. Pergamon Press, Oxford (1963).

18. C. S. Davis, M. L. Barth, B. R. Dudek and R. J. Richardson, in Mechanisms of Toxicity and Hazard Evaluation (Eds. B. Holmstedt, R. Lauwerys, M. Mercier and M. Roberfroid), p. 63. Elsevier/NorthHolland Biomedical Press, Amsterdam (1980).

19. C. S. Davis and R. J. Richardson, Biochem. Pharmac. 36, 1393 (1987).

20. J. Buc, J. Richard and J-C. Meunier, Eur. J. Biochem. 80, 593 (1977).

21. J-R. Garel and R. L. Baldwin, J. molec. Biol. 94, 611 (1975).

22. C. R. Cantor and P. R. Schimmel, Biophysical Chemistry, Part III: The Behavior of Biological Macromolecules, p. 1075. W. H. Freeman, San Francisco (1980).

23. J. Seifert and J. E. Casida, Comp. Biochem. Physiol. 78C, 271 (1984)

24. B. Kohl and H. Sanderman, Fedn Eur. Biochem. Soc. Lett. 80, 408 (1977).

25. A. Helenius and K. Simons, Biochim. biophys. Acta 415, 29 (1975).

26. W. N. Aldridge and E. Reiner, Enzyme Inhibitors as Substrates: Interactions of Esterases with Esters of Organophosphorus and Carbamic Acids, p. 182. North-Holland Publishing, Amsterdam (1972).

27. C. D. Carrington and M. B. Abou-Donia, Biochem. J. 236, 503 (1986) 